\title{
Karl Kraus über Franz Grillparzer
}

\author{
Sigurd Paul Scheichl
}

Im Jänner 1991 wurde der wieder begründete und gleich heftig umstrittene ${ }^{1}$ Grillparzer-Preis an Peter Handke verliehen. Der Preisträger sprach in seinen Dankesworten nicht nur von Grillparzer, sondern auch von Reaktionen auf ihn, zunächst von jenen, die ihn als "Ahnherrn" "beansprucht" haben, aber auch von jenen, für die der Dramatiker ein "Gegenbild" gewesen ist. Und damit meint er vor allem Karl Kraus,

der da wieder einmal aus Scharfsinn kleinlich wird, oder aus Kleinlichkeit scharfsinnig?, und so, wie es eben Scharfsinn und Kleinsinn im Verein anzurichten pflegen, sein Gegenbild - sein Recht - zum Zerrbild, zum Unrecht, zum Verrat seiner Sache an bewährte Wortspielerei mißbraucht: "papierne Ebenheit der Welt Grillparzers", "Halm, dem Verwandten seiner Blutleere" ... - zu welcher Verzerrung auch gehört, es dem Dichter anzurechnen, was seine Ideologen [...] mit ihm anrichten: durch Grillparzer der "Anschluß" ...; und auch gehört, blindlings, reflexhaft, im voraus das Abwerten im Sinn, zu vergleichen: "... Raimund, der der echtere Dichter war", "... Nestroy, an dessen Gebiet außen weder des Meeres noch der Liebe Wellen anschlagen, in jeder Zeile mehr Lyriker, Dramatiker und Epigrammatiker ... als der ganze Grillparzer."

Nein, Karl Kraus, so nicht, nicht dieses sinnlose Entzweien des Zusammengehörenden, nie mehr: Franz Grillparzer und Ferdinand Raimund und Johann Nestroy und, und ..., und, auf deine Weise, auch du, K. K.; $[\ldots]^{2}$

Handkes Rede stellt sich dann übrigens rasch in eine Perspektive, die nicht die von Kraus gewesen ist. Handke nennt sich einen Leser vor allem der Autobiographie und der Tagebücher, einen Bewunderer des Menschen Grillparzer; für Kraus war Grillparzer selbstverständlich vor allem der Autor der von Handke mit keinem Wort erwähnten großen Dramen und daneben gerade noch der Epigramme; die "geistig durchaus über seinen berühmtesten Dramen stehenden" Tagebücher erwähnt er, lobend, überhaupt nur ein einziges $\mathrm{Mal}$ ( $F$ $857-63,1931$, S. 71$)^{3}$, und auch das nur, weil er einer - ihm zugetragenen? -

1. Vgl. Oliver Marchart u. a.: "Grillpatzer". In: Forum (Wien) 38 (1991). Nr. 445447. S. 52-55. (Ebendort S. 55f. ein Bericht über die Preisverleihung: Oliver Marchart, "Bekenntnisse eines Nekrophilen").

2. Peter Handke, "Ich liebe ihn". Peter Handkes Dankrede bei der Verleihung des Grillparzer-Preises 1991 an der Wiener Universität. In: profil 22/4 (21.1.1991). S. $74,76$. 
Tagebuchstelle ( $T g b .3020,1836 ; H K A$ II/10, S. 70) ein Argument gegen Heine und gegen den Journalismus entnehmen zu können glaubt: jener sei bei einem von Rothschilds gegebenen Diner besonders höflich behandelt, "fetiert" worden, damit er niemandem schade. Kraus' Interesse gilt hier also vorwiegend einem inhaltlichen Aspekt, der in seine Polemiken integrierbar ist.

Zurück zu Handke. Dieser untermauert seine Kritik an Kraus' Urteilen über Grillparzer durch verschiedene, alle dem Aufsatz Grillparzer-Feier von 1922 (F 588-94, S. 12-21) ${ }^{4}$ entnommene Zitate, die in der Tat wenig Begeisterung für den Dichter verraten. Diese negativen Urteile der Fackel über Grillparzer sind umso bemerkenswerter, als sich Kraus ja sonst durchaus zu literarischen Traditionen bekannt und sich sogar in einem Gedicht ausdrücklich als einen "Epigonen" (Bekenntnis, S 9, S. 93) ${ }^{5}$ bezeichnet hat. In der österreichischen Literatur des 19. Jahrhunderts schätzte er besonders Nestroy, als dessen Wiederentdecker und Erben er sich fühlte, und Raimund; auch Stifter hat er zwar nicht sehr häufig, aber stets mit dem Ausdruck größter Bewunderung genannt. ${ }^{6}$ In diesem positiven Verhältnis zur literarischen Tradition ${ }^{7}$ spielt Grillparzer nun so gut wie keine Rolle; eine wesentlich geringere auch als der von Kraus zwar ebenfalls eher kritisch beurteilte, aber doch sehr häufig zitierte Schiller ${ }^{8}$, von dem er gegen Ende seines Lebens sogar einige Balladen in die Vorlesungsprogramme aufnahm. ${ }^{9}$ Zurecht ist Werner Kraft in seinem Buch über

3. Die Zeitschrift Die Fackel wird fortlaufend im Text mit der Sigle $F$ zitiert; wie üblich schließen sich daran die Angabe der Heftnummer(n) und des Erscheinungsjahres sowie der Seite an.

4. Die erste Stelle - bei Handke, S. 74, "der Taferl-Klassiker Grillparzer" - ist ungenau zitiert; alle Stellen, die Handke anführt, finden sich in F 588-94 auf S. 13.

5. Von Kraus in seine Bücher aufgenommene Werke werden zitiert nach der Ausgabe: Karl Kraus, Schriften. Hrsg. von Christian Wagenknecht. 12 Bände. Frankfurt/M. 1986-1989; mit der Sigle $S$ und Angabe des Bandes.

6. Sigurd Paul Scheichl, "Karl Kraus und Adalbert Stifter". In: Johann Lachinger (Hrsg.), Sammelband zur Stifter-Rezeption. Linz 1992 (im Druck).

7. Kurt Krolop, ''Ahnenwerthes Ahner' - Zur Genesis und Funktion der Traditionswahl bei Karl Kraus" (1979). In: KK: Sprachsatire als Zeitsatire bei Karl Kraus. Berlin (DDR) 1987 (=Literatur und Gesellschaft). S. 155-176, 321325; vgl. auch andere Beiträge in diesem Band. Ferner Werner Kraft, Das Ja des Neinsagers - Karl Kraus und seine geistige Welt. München 1974.

8. Dazu Kurt Krolop, "'Die Berufung auf Schiller wird zur Gänze abgewiesen' Schiller-Bezüge der Dritten Walpurgisnacht im Lichte der Fackel". In: KK: Sprachsatire (Anm. 7), S. 231-251, 343-347.

9. Dazu Christian Wagenknecht, "Lyrik der Deutschen" - für seine Vorlesungen ausgewählt von Karl Kraus. In: Karl Kraus in neuer Sicht. Londoner KrausSymposium. Sigurd Paul Scheichl, Edward Timms (Hrsg.). München 1986. S. 143157. Hier: S. 151f. 
Kraus' Bejahung der literarischen Tradition auf Grillparzer überhaupt nicht eingegangen. ${ }^{10}$

Es scheint gerade in Hinblick auf die Traditionsverbundenheit seines Werkes sinnvoll, den Urteilen Kraus' über den Dramatiker nachzugehen und herauszufinden, welche Kriterien für seine Ablehnung des Dichters maßgebend gewesen sein mögen.

Zunächst einmal kann man mit einer gewissen Sicherheit annehmen, daß Kraus das Werk Grillparzers einigermaßen gut gekannt hat. Er war ja eben zu dem Zeitpunkt noch Gymnasiast, als der Unterrichtsminister für den Deutschunterricht an österreichischen Gymnasien eine stärkere Berücksichtigung von Literatur aus Österreich und insbesondere von Grillparzer vorgeschrieben hat. ${ }^{11}$ So fand auch an seinem Gymnasium am 15. Januar 1891 "anläßlich des hundertsten Gedächtnistages der Geburt Grillparzers" "für die Schüler des Obergymnasiums eine Festfeier" statt, bei der auch der "Vortrag von Dichtungen Grillparzers seitens der Schüler der VII. Klasse" Járay, Kraus und Pollak erfolgte. ${ }^{12}$ Spät ( $F$ 657-67, 1924, S. 200) erwähnt Kraus diese Veranstaltung selbst, bei der ihm die Aufgabe zugefallen sei, Ottokar von Hornecks Lobpreis auf Österreich zu rezitieren:

wozu man nur entweder Schiller zitieren kann, der das Glück die Gaben ohne Wahl verteilen läßt, oder einen der neueren Autoren, die den Fall bündiger mit dem Wort "ausgerechnet" formulieren würden.

Diese Bemerkung bezieht sich darauf, daß Kraus als Verfasser der Letzten Tage der Menschheit in der Öffentlichkeit nach dem Ersten Weltkrieg nicht gerade den Ruf hatte, ein Lobredner Österreichs zu sein.

$\mathrm{DaB}$ Kraus in der Schule Grillparzer gelesen hat, steht also, anders als im Fall Stifter ${ }^{13}$, fest. Wie ausgedehnt seine Grillparzer-Lektüre gewesen ist, muB hingegen offen bleiben; auf allzu viele Werke des Dichters geht er jedenfalls nicht ein, und schon gar nicht in einem Ausmaß, aus dem eine wirkliche Auseinandersetzung mit dem jeweiligen Werk sich erschließen ließe.

Ohne Zweifel hat Kraus auch auf den Wiener Theatern GrillparzerAufführungen gesehen, doch kommen in seinen immer wieder niedergelegten

10. Kraft (Anm. 7); auch in Krolop, Sprachsatire (Anm. 7) sind die Hinweise auf Grillparzer spärlich.

11. Klaus Zelewitz, "Schiller oder Grillparzer? Bemerkungen zum Gymnasialunterricht in der österreichischen Reichshälfte der Doppelmonarchie um 1900." In: Germanica Wratislaviensia 77 (1987). S. 178-198. Hier: S. 181f.

12. 17. Jahresbericht über das $k$. k. Franz-Joseph-Gymnasium in Wien. 1890/91. Wien 1891. S. 15. Zitiert nach Gilbert J. Carr: Hinweis 173: "Kraus' erste Grillparzer-Feier." In: Kraus-Heft 41 (1987). S. 8.

13. Vgl. Scheichl (Anm. 6). 
Äußerungen über die großen Wiener Schauspielerinnen und Schauspieler des ausgehenden 19. Jahrhunderts Grillparzer-Rollen so gut wie überhaupt nicht vor. Nur einmal, ohnehin in der seine Distanz zu Grillparzer am deutlichsten artikulierenden Satire Grillparzer-Feier gesteht er ganz allgemein den "Grillparzerdramen" zu: "[...] so außerordentlich sie zur Unterlage einer hohen Schauspielkunst taugen mochten [...]" (F 588-94, 1922, S. 12), nennt aber auch hier keine Schauspielernamen; die Stelle ist auch insofern kein Kompliment für Grillparzer, als Kraus zwischen Literatur und Theater stets streng unterschied und die Eignung gerade von schlechten Bühnentexten für große Schauspieler immer wieder unterstrich ${ }^{14}$ Allein in einem der letzten Hefte der Fackel erwähnt Kraus 1935 in einer Anmerkung zu Erinnerungen an die Schauspielerin Zerline Gabillon ${ }^{15}$ auch einige wenige, nicht besonders gewichtige Grillparzer-Rollen, die deren Mann Ludwig Gabillon gespielt hatte ( $F$ 912-15, 1935, S. 23f.).

Angesichts der Bedeutung, die das Wiener Theater in "jenen figürlichen Achtziger Jahren" (Jugend, S 9, S. 206) für Kraus hatte, läßt sich aus dieser Tatsache wohl der Schluß ziehen, daß Grillparzer den jungen Karl Kraus nicht besonders beeindruckt haben dürfte; allerdings ist Grillparzer gerade am Burgtheater, das Kraus in besonderem Maße geprägt hat, keineswegs besonders häufig gespielt worden. ${ }^{16}$ Daraus mag sich auch das Fehlen von Erinnerungen an Schauspieler in Grillparzer-Rollen zum Teil erklären. In den gesammelt vorliegenden frühen Aufsätzen und Rezensionen ${ }^{17}$ fällt der Name Grillparzer ebenfalls so gut wie nie und schon gar nicht im Zusammenhang einer Beschäftigung mit einem bestimmten Werk, während sich die Jung-Wiener Zeitgenossen Kraus' durchaus immer wieder auf Grillparzer bezogen haben. ${ }^{18}$

Dennoch war Grillparzer auch für Kraus so etwas wie selbstverständlicher

14. Vgl. z.B. "Girardi und Kainz", F 254-55 (1908) S. 4-7, besonders S. 5f. oder "Die Literaturlüge auf dem Theater", F 457-61 (1917) S. 53-57, besonders S. 57.

15. In dem Gedicht "Liebeserklärung an Zerline Gabillon" nennt Kraus diese, 1892 gestorbene, Schauspielerin die "von allen Rahels liebenswerteste" ( $S$ 9, S. 606); diese Stelle kann sich aber nicht auf die Jüdin von Toledo beziehen, da Zerline Gabillon in dieser nur die Rolle der Esther gespielt hat (und das schon 1873, also vor Kraus' Zeit).

16. Vgl. die Listen der gespielten Stücke in: 175 Jahre Burgtheater 1776-1951. Wien o. J. (1955); zwischen 1879 und 1902 sind nur sechs Grillparzer-Dramen auf dem Programm gestanden.

17. Karl Kraus, Frühe Schriften. 2 Bände. Hrsg. von Johannes J. Braakenburg. München 1979.

18. Das Register von: Gothart Wunberg (Hrsg.), Das Junge Wien. Österreichische Literatur- und Kunstkritik 1887-1902. 2 Bände. Tübingen 1976, weist insgesamt sechsundachtzig Grillparzer-Erwähnungen in den dort zusammengetragenen Artikeln aus Zeitungen und Zeitschriften nach. 
Bildungsbesitz. Er wird in der Fackel von 1899 bis 1936 immerhin einhundertfünfzehn $\mathrm{Mal}$ erwähnt ${ }^{19}$, wobei, wie immer bei Kraus, auch Stellen berücksichtigt sind, in denen er andere zitiert. Immerhin ist der wenig geschätzte Grillparzer so in der Fackel viel mehr präsent als der bewunderte (aber wohl nicht besonders gut gekannte) Stifter, der auch erst 1911 in der Fackel erwähnt wird und insgesamt nur sechzehn Mal aufscheint; Grillparzers Name fällt dagegen schon 1899 im ersten Heft von Kraus' Zeitschrift $(F 1,1899$, S. 26).

Bereits diese Stelle zeigt Kraus als einen, der gewisse Aspekte des Grillparzerschen Werkes zitierend abrufen kann: hier bezieht er sich auf Der Traum ein Leben, um eine Stelle in einem neuen Hofmannsthal-Stück durch Kontrastierung als fragwürdig hinzustellen. ${ }^{20}$ Andere Grillparzer-Zitate werden folgen, wenn auch nicht besonders viele. Mag sein, daß da das eine oder andere noch ermittelt werden kann; so hat jüngst Gustav Kars, noch aus dem Wissen eines österreichischen Gymnasiasten der Vorkriegszeit - das aber Kraus bei seinen Leserinnen und Lesern vorausgesetzt hat ${ }^{21}$ - eine Stelle aus dem wichtigen programmatischen Aufsatz Bekenntnisse von 1905 (F 185, S. 8):

Die Zeiten sind vorbei, wo mich die Kunde stolz machen konnte, daß liberale Väter ihren schwangeren Frauen den Anblick der roten Hefte entzogen und den schon gezeugten Kindern zuriefen: Hütet Euch, je zu werden diesem gleich!

als Zitat aus der Ahnfrau erkannt. ${ }^{22}$ (Daß Kraus bei seinen Leserinnen und Lesern zurecht solche Grillparzer-Kenntnisse voraussetzte, geht unter anderem daraus hervor, daß der Verfasser einer der letzten Polemiken gegen Kraus, die zu dessen Lebzeiten erschienen sind, mit deutlicher Anspielung auf den Räuber aus

19. Für die Erfassung solcher Stellen ist Franz Ögg, Personenregister zur Fackel von Karl Kraus. München 1977, ein unentbehrliches Hilfsmittel. Die Zahlen, die ich angebe, stimmen mit den seinen nicht ganz überein, da einige Irrtümer zu korrigieren waren. Ferner habe ich seine Liste durch einige im Index von Gustav H. und Franz H. Bäuml, "Namenverzeichnis zu Karl Kraus' DIE FACKEL." In: Modern Austrian Literature 6 (1973). Heft 1/2. S. 151-182. Heft 3/4. S. 139-160; 7. 1974. Heft 1/2. S. 141-173; 8. 1975. Heft 3/4. S. 197-221; 9. 1976. Heft 1. S. 44-83. Heft 2. S. 10-44, angeführte Stellen ergänzt. Schließlich habe ich einige bei $O_{g g}$ angeführte Namensnennungen als zu peripher in die Statistik nicht aufgenommen.

20. Vgl. auch die recht genaue Anspielung auf dieses Stück in $F$ 292, 1909, S. 21.

21. Vgl. über die Zitate bei Kraus Sigurd Paul Scheichl, "Von der Männerschwäche und der Weihe der Kraft. Über praktische Probleme des Kommentierens von Karl Kraus." In: Mitteilungen aus dem Brenner-Archiv 9 (1990). S. 28-37. Hier: S. $35 \mathrm{f}$.

22. Gustav Kars, "Hinweis 258: Grillparzer." In: Kraus-Heft 57 (1991). S. 8. 
der Ahnfrau das Pseudonym "Jaromir" gewählt hat. ${ }^{23}$ )

Eine wichtige und bekannte Grillparzer-Anspielung ist der Titel der Satire Der Traum ein Wiener Leben von 1910 (F 307-08, S. 51-56). Andere Erwähnungen und Anspielungen dieser Art kommen vor, wobei sie sich im großen und ganzen auf Ahnfrau, König Ottokars Glück und Ende, Der Traum ein Leben - auf das auch Grillparzer-Feier relativ ausführlich zu sprechen kommt (F 588-94, 1922, S. 13) und das Kraus besonders gut gekannt zu haben scheint und Weh dem, der lügt! reduzieren lassen. Ob Kraus die anderen Stücke nicht, mindestens nicht gut gekannt, ob er sie weniger (oder gar nicht) geschätzt hat oder ob sie überhaupt nur in geringerem Maß Bestandteil des Bildungsgutes der Wiener Bourgeoisie gewesen sind, wage ich nicht zu entscheiden. Ein Grund für das Fehlen insbesondere der Griechen-Dramen Grillparzers in dieser Liste könnte sein, daß gerade diese in den für den Satiriker prägenden achtziger und neunziger Jahren kaum auf dem Spielplan des Burgtheaters gestanden sind. ${ }^{24}$

Auffällig ist das zweimalige Vorkommen eines Grillparzer-Zitats in Kraus' Glossen in den zwanziger Jahren. In einer Zeit zunehmender Kritik an der Wiener Sozialdemokratie stellt Kraus in der Glosse Je nachdem (F 759-65, 1927, S. 9f.) eine sozialdemokratische und eine christlichsoziale Darstellung von Vorfällen bei Wiener Wahlen einander gegenüber und erinnert daran, daß in der Zeit christlichsozialer Mehrheiten in der Arbeiter-Zeitung etwa das zu lesen war, was nun, in der Ära des 'Roten Wien', die christlichsoziale Reichspost schrieb.

[...] "weil", nach Grillparzer, "was Brot in einer Sprache, Gift heißt in des andern Zunge, und der Gruß der frommen Lippe Fluch scheint in dem fremden Ohr"; (das ruft diesen Schmerz empor).

Wenig später, 1929 ( $F$ 820-26, S. 148) gebraucht Kraus dieses Zitat aus Der Traum ein Leben (I. Akt; V. 253ff.) ${ }^{25}$ noch einmal, in ganz ähnlichem Zusammenhang als Angriff auf den Stil der Auseinandersetzungen zwischen den Parteien in der Ersten Republik.

Damit setzt er, obwohl er hier den Namen des Autors nennt, doch GrillparzerKenntnisse bei den Leserinnen und Lesern seiner Zeitschrift voraus. Das gilt erst recht beim vereinzelten Einbau von Zitaten aus Grillparzer-Epigrammen in seine eigenen Texte, vor allem in den folgenden Aphorismus aus Nachts $(S 8, S$. 370):

23. Jaromir [= Robert Ungar], Letzten Endes. Eine Studie über Karl Kraus. Wien o. J. [recte: Brünn, Auslandsbüro österreichischer Sozialdemokraten 1935].

24. Vgl. Anm. 16.

25. "Das ruft diesen Schmerz empor" ist der letzte Vers des Grillparzer-Zitats. 
Hast du vom Kahlenberg die Stadt dir nur besehn, so wirst du, was ich schrieb und was ich bin, verstehn!

Kraus schreibt die Stelle, obwohl er den Grillparzerschen Reim ${ }^{26}$ beibehält, als Prosa. Er ersetzt Grillparzers "besehen" interessanterweise durch das 'poetischere' "besehn", ebenso "verstehen" durch "verstehn"; ferner macht er, vielleicht aus Gründen des Parallelismus, aus "wer ich bin" "was ich bin". Allerdings müssen diese Veränderungen gar nicht auf Kraus zurückgehen; womöglich hat er eine ungenaue Ausgabe benützt oder folgt einem Zitat des Epigramms an einer heute nicht mehr ermittelbaren Stelle, am ehesten in einer Wiener Zeitung. ${ }^{27}$ Die entscheidende Veränderung ist ohnehin eine andere: wo Grillparzer vom "Land" rings um Wien spricht, schreibt Kraus "die Stadt dir nur". Der Text, der in einer Aphorismenfolge mit dem Titel "Wien" steht, verkehrt sich dadurch von Grillparzers Bekenntnis zu seiner Verwurzelung in der niederösterreichischen Landschaft zu einem aggressiven Bekenntnis des Satirikers, als der Kraus diesen Aphorismus ja geschrieben hat, zu den Wurzeln seines Hasses in der von ihm negativ erfahrenen Stadt Wien. Die Schärfe des Aphorismus ist nur erkennbar, wenn man den Grillparzerschen Prätext mitbedenkt, dessen Kenntnis der Satiriker somit voraussetzt. Woher Kraus die Verse gekannt hat, muß offen bleiben; sie mögen durchaus im damaligen Wien öfter zitiert worden sein. ${ }^{28}$

Mit ganz anderer Funktion spielt Kraus auch in Grillparzer-Feier (S. 13) auf diese Stammbucheintragung an:

[...] und die psychologischen Schwätzer wie jener Bahr mögen [...] aus der Ubereinstimmung des persönlichen und des ethnischen Wesens zugleich Ehre für das Land und für seinen Dichter aufheben - die mäßige Höhe, in der man vom Kahlenberg sich jenes besieht, dürfte auch diesem ein für allemal den Rang bestimmen, $[\ldots]$

Ein zweites, wesentlich weniger deutlich ausgeprägtes Beispiel für eine solche Integration eines Grillparzer-Wortes in einen Aphorismus von Kraus findet sich noch in der letzten, 1919 in der Fackel erschienenen, aber in keinen Aphorismenband aufgenommenen Aphorismenkette ( $F$ 508-13, 1919, S. 80; $S$ 8, S. 463):

Humanität und Bestialität: wann wird man endlich jene mit dem Geschmack des

26. Es handelt sich bei Grillparzer um die zweite Fassung einer Stammbuch-Eintragung von etwa $1844 ; H K A \mathrm{I} / 12$, S. 153.

27. Vgl. das Zeitungszitat des Epigramms, aus dem Abend, allerdings von 1922, mit eben diesen Abweichungen in F 588-94, S. 19.

28. Vgl. Anm. 27. 
Hasses, diese mit dem der Liebe aussprechen? Zerfleischt ein Löwe seinen Nebenlöwen?

Schwer vorstellbar, daß Kraus hier nicht an Grillparzers vielzitierten Weg der neuern Bildung von 1849 gedacht haben sollte (HKA I/12, S. 213), obwohl er die Nationalität beiseite läßt. Allerdings gibt der neuere Satiriker dem Aphorismus eine ganz andere Wendung, die durchaus auch ohne Kenntnis des Prätextes, wenn er denn überhaupt einer ist, verständlich ist.

Weitere Beispiele für intertextuelle Bezüge zwischen Kraus- und GrillparzerTexten mag es noch geben; sehr zahlreich sind solche Stellen gewiß nicht. Zu zeigen war hier ja nur, daß Kraus Grillparzer gekannt und daß er bei seinem Publikum mit einer gewissen Grillparzer-Kenntnis gerechnet hat.

In diesem Sinne beruft sich Kraus jahre-, ja jahrzehntelang auf Grillparzer als einen selbstverständlichen Bildungsbesitz, ohne wirklich die Frage nach Grillparzers Bedeutung zu stellen. Daß solche Bildungsvorstellungen für Kraus trotz aller Abneigung gegen Bildungsdichter ( $F$ 657-67, 1924, S. 200, auf Grillparzer bezogen ${ }^{29}$ ) von Bedeutung gewesen sind, kann ich hier im einzelnen nicht begründen; es wird sich aus den folgenden Zitaten ohnehin von selbst ergeben.

Ein Hauptmotiv der Grillparzer-Erwähnungen ist die Kontrastierung zwischen den leichten Erfolgen heutiger Schriftsteller und den Schwierigkeiten, die ein Autor vom Range Grillparzers hatte; darüber hinaus der Gegensatz zwischen einer durch die Zeitungen taxfrei an mediokre Gestalten verliehenen Popularität und dem zurückgezogenen Dasein des Autors.

So erinnert Kraus 1901 Hermann Bahr, der gerade wieder eifrig seine JungWiener Freunde gelobt hatte, daran, "dass vor Schnitzler Grillparzer in ganz Deutschland gespielt und vor Klimt Makart in der ganzen Welt bewundert und dass beide trotz ihren ausländischen Triumphen hierzulande nicht angegriffen wurden" (F 73, S. 9). Ähnlich heißt es 1912 ( F 354-56, S. 52) über Bahr:

Er ist zwar schon fünfzig, findet aber noch immer Käufer und wird demnächst Lobsprecher seiner Reize finden, wie sie Grillparzer im gleichen Alter nicht gefunden hat, wiewohl er sich auch für den höchsten Betrag zu nichts hergegeben hätte.

Auch gegen Vergleiche mit dem Schriftsteller Hans Müller glaubt Kraus Grillparzer schützen zu müssen (z.B. $F$ 445-53, 1917, S. 60f.), obwohl sie zunächst wegen der historischen Themen von Müllers Stücken nahe zu liegen scheinen und weil Grillparzer und Hans Müller gemeinsam auf dem Programm einer Gastspielreise des Burgtheaters in die Schweiz standen ( $F$ 454-56, 1917, S.

29. Die Stelle ist unten zitiert. 
13). Noch im Heft, in dem die kritische Grillparzer-Feier steht, amüsiert sich Kraus darüber, daß Müller in den Zeitungen ausdrücklich als Teilnehmer der Grillparzer-Feier des Journalistenvereins Concordia genannt wird ( $F$ 588-94, 1922, S. 40). Es sei noch ein Vergleich mit Hofmannsthal aus den Kriegsjahren (F 423-25, 1916, S. 51) ${ }^{30}$ zitiert:

Herr Hofmannsthal, der vom Vaterland erwartet, daß es ihn nicht rufe, wenn er von Schlachtenruhm träumt, aber wenn er erwacht, ihm Grillparzers Ehren erweise [...]

Diese Technik findet sich nach der kritischen Grillparzer-Feier nur noch selten, sie wird aber immerhin 1931 ( $F$ 852-56, S. 71) gegen Wildgans angewendet:

Kann der Osterreicher Wildgans wirklich als Fünfzigjähriger den wochenlangen Schall der Salven hinnehmen, von denen das ganze Leben eines Grillparzer unberührt geblieben ist, als dessen Erben er sich doch fühlt und den er gewiß für keinen Taferlklassiker hält? ${ }^{31}$

Mehrfach, zuletzt auch in der stilisierten Form eines Aphorismus vergleicht Kraus schließlich die von der Presse dem eitlen Caféhaus-Besitzer Ludwig Riedl erwiesenen Ehren mit den Nicht-Ehrungen Grillparzers: "Immerhin bleibt die Frage offen, ob [...] sich dreihundert Vertreter des Wiener Bürgertums um die Ehre beworben haben, Grillparzer zu seinem Geburtstag zu gratulieren und ihre Namen bei diesem Anlasse in die Zeitung zu bringen." ( $F$ 267-68, 1908, S. 47). ${ }^{32}$

Diese Stellen sind wohl auch nicht zu überschätzen, wie schon der Vergleich mit Makart andeutet. Es geht hier kaum um Grillparzer, schon gar nicht um eine substantielle Beschäftigung mit seinem Werk; vielmehr ist der Name - immerhin - als eine Chiffre mit der Bedeutung 'großer, verkannter Mann' eingesetzt, um Kontraste zu unterstreichen. Im Sinne von Kraus' Methode der "immanenten Kritik"33 ist darüberhinaus nicht auszuschließen, daß Kraus diesen Namen nicht deshalb gewählt hat, weil Grillparzer für ihn selbst wichtig ist, sondern weil der Autor im österreichischen Bürgertum so hohes Ansehen genoß; Kraus mißt die

30. Kraus spielt hier selbstverständlich auf Hofmannsthals 1915 erschienene Schrift "Grillparzers politisches Vermächtnis" an.

31. Das Wortspiel "Taferlklassiker" setzt die Kenntnis des Austriazismus Taferlklasse' mit der Bedeutung '1. Grundschulklasse' voraus. Kraus zitiert sich hier, wie oft, selbst, nämlich eben Grillparzer-Feier, S. 13.

32. Vgl. auch $F$ 345-46 (1912), S. 53; $F$ 445-53 (1917), S. 7.

33. Theodor W. Adorno, "Sittlichkeit und Kriminalität. Zum elften Band der Werke von Karl Kraus." In: TWA: Gesammelte Schriften 11: Noten zur Literatur. Frankfurt/M. 1974. S. 367-387. Hier: S. 370. 
Riedl-Gratulanten somit nicht an seinen, sondern an den (mindestens vorgeschützten) Wertvorstellungen seiner Zeitgenossen.

Mit gleicher Selbstverständlichkeit setzt Kraus voraus, daß der Gebildete über Grillparzer Bescheid weiß. Er höhnt so über das Neue Wiener Journal, das glaubt, Ferdinand von Saar sei als erster Schriftsteller ins Herrenhaus berufen worden, während diese Ehre doch Grillparzer zuteil geworden war ( $F$ 208, 1906, S. 24). Und noch viel später, 1923, verhöhnt er, gerade hier ganz gewiß in der Verfahrensweise der 'immanenten Kritik', den katholischen Publizisten Joseph August Lux, weil gerade er ein Schiller-Zitat Grillparzer zugeschrieben hat ( $F$ 622-31, 1923, S. 154f.).

Vereinzelt bleibt eine gegen den Theaterkritiker Schütz gerichtete Stelle, in der dessen Unverständnis für moderne Dramatik, insbesondere für Wedekind, angeprangert wird. Schütz ist für Kraus ein "Repräsentant jener Kreise, die [...] sich einst auch über die Zumutungen der Grillparzer, Hebbel und Ibsen erhaben gefühlt haben." ( $F$ 189, 1905, S. 26). Der Vergleich ehrt Grillparzer; er ist freilich zu isoliert, um daraus Wertschätzung Kraus' für Grillparzer als einen Erneuerer des Dramas im 19. Jahrhundert wenigstens in dieser Periode der Fackel abzuleiten. Auch hier steht der Name wohl in polemischem Zusammenhang als Chiffre im skizzierten Sinn.

Sympathie für den Autor läßt sich am ehesten den wenigen Stellen entnehmen, an denen Kraus ihn gegen seine eigenen Gegner verteidigt. Er will der Psychoanalyse die Beschäftigung mit Grillparzer verbieten $(F 387-88,1913$, S. 21); vor allem auch wehrt er sich dagegen, daß der Liberalismus versucht, Grillparzer als einen der Seinen zu vereinnahmen: so scheint der schon genannte Schütz versucht zu haben, Grillparzers Medea in die Diskussion um den "Sprachenstreit" hereinzuziehen ( $F$ 40, 1900, S. 25) oder den Autor aufgrund ebenfalls von Medea und von Weh dem, der lügt! als einen Kronzeugen gegen den Antisemitismus erscheinen zu lassen ( $F$ 150, 1903, S. 7f.). Und ein Aphorismus von 1912 (F 360-62, S. 15) lautet:

Der Liberalismus beruft immer, wenn einer der Seinen stirbt, das Schicksal Grillparzers und beschuldigt Österreich. Als ob heute der Dichter am Staat und nicht an der Welt litte. Und als ob Grillparzer, wäre er heute gestorben, sich durch Lieferung von Feuilletons für die vaterländische Unbill entschädigt hätte.

Damit ist Kraus' Grillparzer-Bild bis in die frühen zwanziger Jahre einigermaßen umrissen: kühler Respekt vor dem Bildungsgut, kaum mehr als Interesselosigkeit, freilich auch kaum eine Spur von Ablehnung.

Diese Einstellung Kraus' zu Grillparzer macht es recht unwahrscheinlich, daß sein Urteil über diesen bei seiner einzigen persönlichen Begegnung mit Wittgenstein eine Rolle gespielt haben könnte. Offensichtlich vor 1914 hatte Paul 
Engelmann Kraus und Wittgenstein einander vorgestellt; das Zusammentreffen der beiden soll allerdings mit Streit über einen Dichter geendet haben, den der Philosoph schätzte, der Satiriker aber nicht. Reinhard Merkel, der diese Episode erfahren und berichtet hat ${ }^{34}$, hat nun vermutet, daß ein Gespräch über Grillparzer diesen Streit provoziert haben könnte. Diese Vermutung kann im Licht der vorgestellten Äußerungen Kraus' über Grillparzer schwerlich überzeugen; vor 1914 scheint der Satiriker über diesen nicht so viel nachgedacht zu haben, daß er eine Auseinandersetzung über ihn hätte führen mögen oder können.

Nur an insgesamt drei von vielen Stellen läßt sich ein Anflug von Kritik erkennen. 1910 zitiert Kraus das satirische Gedicht Der Henker hole die Journale [...] (1844; HKA I/11, S. 191), durchaus zustimmend, indem er ihm die aktualisierende Überschrift Dem internationalen Preßkongre $\beta$ gibt (F 303-04, 1910, S. 25). In einer Notiz dazu nennt er es allerdings ein "Gedicht, das manche Dramen Grillparzers aufwiegt"35, eine Formulierung, die doch eine gewisse Distanz gegenüber dem dramatischen Werk impliziert.

Die beiden anderen Stellen betreffen König Ottokars Glück und Ende und sein patriotisches Pathos, das Kraus vielleicht wirklich schon seit der Gymnasialzeit suspekt gewesen ist. Den Publizisten Friedjung, der sich in einer politischen Affäre sehr ungeschickt mit patriotischen Phrasen profiliert hatte, verspottet Kraus wegen seines an den Schauspieler Schreiner erinnernden "sonoren Organs", das sich besonders für die Rede des Ottokar von Horneck eigne ( $F$ 293, 1909, S. 16), mit dem er Friedjung darüberhinaus in eins setzt. In einer Kriegsglosse über den Bankier Sieghart findet sich eine ähnliche despektierliche Bemerkung über die Atmosphäre des Ottokar (F 445-53, 1917, S. 132).

1922 sind dann plötzlich nur noch diese kritischen Ansätze da. In GrillparzerFeier (F 588-94, 1922, S. 12-21) und an einer Reihe von - im folgenden noch erwähnten - späteren Stellen, in denen Kraus mehr oder minder seine Urteile aus diesem Text bestätigt oder auf sie anspielt, wertet er den bisher mit distanzierter Achtung erwähnten Autor eindeutig $\mathrm{ab}$, bis zu einem Vergleich mit Friedrich Halm, "dem Verwandten seiner Blutleere" (S. 12), der später durch die Gleichordnung Grillparzers mit Anzengruber noch verschärft wird ( $F$ 676-78, 1925 , S. $42 ; F \quad 679-85,1925$, S. 82). Die griffigen Formulierungen des satirischen Artikels von 1922 bestimmen unser Bild - wie das Handkes - von Kraus' Einstellung zu Grillparzer.

Um diese Wendung zu verstehen, ist zunächst auf den Anlaß dieses Fackel-

34. Reinhard Merkel, "'Geistige Landschaft mit vereinzelter Figur im Vordergrund': Ludwig Wittgenstein." In: Merkur 38 (1984). S. 659-671. Hier: S. 668f. Merkel hat diese Information von Werner Kraft erhalten, dem wiederum Engelmann den Vorfall erzählt haben soll.

35. An dieser Stelle erwähnt er auch die von ihm wohl verwendete Gesamtausgabe bei Cotta von 1887 als Quelle für das Zitat. 
Beitrags einzugehen. 1922 wurde Grillparzers fünfzigster Todestag begangen, verbunden mit der Öffnung seines Nachlasses; es gab offizielle Veranstaltungen und zahlreiche Artikel in der Presse. Kraus hatte gegen derartige Jubiläen seit jeher Bedenken; schon 1899 ( $F$ 15, S. 11-16) kritisiert ein Mitarbeiter die Art, in der das Goethe-Jubiläum begangen wurde; und der Titel Grillparzer-Feier ist eine Art Selbstzitat, nämlich des Titels von Kraus' Polemik gegen Schnitzler, SchnitzlerFeier von 1912 (S 3, S. 161-172), die sich ebenfalls weniger gegen den geehrten Autor als gegen die Gratulanten zu dessen fünfzigstem Geburtstag richtete.

Der Protest gegen die 'Schmach, die Unwert schweigendem Verdienst erweist $^{\prime 36}$, indem er den lauten Unverdienten zujubelt, der Einspruch gegen die "unabänderlich falsche Maßverteilung, mit der sich das Urteil der Welt und Nachwelt, jenes vom Journalismus, dieses von der Literaturgeschichte beirrt, an den geistigen Werten vergreift" (S. 12), ist auch in Grillparzer-Feier unüberhörbar, schon dadurch, daß sich eigentlich nur ungefähr ein Drittel des Textes mit dem gefeierten Dichter, der Rest mit Verfassern von Texten zum Jubiläum beschäftigt, vornehmlich mit Thomas Mann - dem Kraus immerhin zubilligt, "bei aller Überschätzung des Gesamtwerks" in der Hervorhebung "vereinzelter Schönheiten" "ein richtiges Urteil [zu] bewähren" (S. 14) - und "unserem Wildgans" (S. 15), dem er gar nichts zubilligt, dafür aber eine Reihe mißlungener Verse im Prolog zur Grillparzer-Feier nachweist. Die Art des Feierns, zu dem obendrein auch ein Hermann Bahr, Kraus' alter Feind, beitrug (S. 13), löst mehr als der Gefeierte selbst den Protest des Satirikers aus; wie auch die Polemik gegen Heine eine Reaktion auf die "Folgen" und auf den liberalen HeineKult ist, wie auch Kraus' Nestroy-Kult durch die Fehlurteile der Literaturgeschichte wesentliche Impulse empfangen hat.

Bei diesem Protest gegen Grillparzer sind ferner die konkreten Zeitumstände des Jahres 1922 zu beachten. Das alte Österreich, das in Grillparzer eine Art Staatsdichter erblickt hatte oder als dessen Staatsdichter man Grillparzer auffassen konnte, war 1918, unter dem Beifall von Kraus, zusammengebrochen; Österreich war für den Satiriker als der Staat, der vor allem Schuld am Weltkrieg trug, problematisch geworden, und der Versuch, "aus der Übereinstimmung des persönlichen und des ethnischen Wesens zugleich Ehre für das Land und für seinen Dichter" aufzuheben (S. 13), war in Kraus' Augen für Grillparzer geradezu diffamierend; da er das "ethnische Wesen" Österreichs jetzt aufs heftigste angriff, mußte er auch den Rang des "vaterländischen Dichters" (S. 21) in Zweifel ziehen. Nach der Erfahrung des Ersten Weltkriegs, in dem Kraus ein unerbittlicher und unerschrockener Kritiker der staatlichen Gewalt gewesen war, flößte ihm "ein vor allen Gewalten des Lebens und des Staates unterduckendes Gemüt" (S. 13) wie das

36. Dieses Zitat aus dem großen Monolog Hamlets ist bei Kraus nicht selten; vgl. z. B. die Stelle im Gedicht Nach zwanzig Jahren, S 9, S. 293. 
Grillparzers Verachtung ein. Daß Kraus bei diesem Urteil, wie auch sonst, durchaus unhistorisch dachte, sei immerhin angemerkt. Ganz in diesem Sinn erklärt auch Rudolf Kassner im Rückblick die Entstehung von so "etwas wie einer Fronde gegen den vaterländischen Dichter" im Umfeld von Kraus mit dessen "Jubelrufen" über den Untergang Österreich-Ungarns. ${ }^{37}$

Verstärkt wurde dieses Urteil durch eine gewisse konservative Schlagseite der Grillparzer-Ehrungen, bei denen auch die sozialdemokratisch verwaltete Stadt Wien auf einen Festprolog von Anton Wildgans nicht verzichten zu kőnnen glaubte, ${ }^{38}$ durch das Vorhandensein eines "mageren Bedürfnisses, das, auf Stoff und Gesinnung beschränkt, selbst heute noch an Radetzky-Verklärungen sich befriedigt und in all seinen christlich-deutschen Belangen auch mit einem Taferlklassiker vorlieb nehmen würde." (S. 13)

Schließlich argumentiert Kraus auch mit dem durch viele literaturgeschichtliche Publikationen geisternden Klischee von Grillparzer als dem 'dritten deutschen Klassiker' nach Goethe und Schiller, das "vornehmlich aus dem Bedürfnis Österreichs nach einem Klassiker entstanden" sei (S. 12). In die Ablehnung dieser Vorstellung fließen ebenfalls nicht nur literaturkritische, sondern auch politische Gesichtspunkte ein. Denn Kraus, der schon aufgrund der Erfahrungen mit dem Bundesgenossen während des Ersten Weltkriegs von Anfang an ein Gegner des 'Anschlusses' gewesen ist, sieht in der Zuweisung dieser Rolle an Grillparzer einen Teil von Österreichs damaligem Bemühen, "den unmittelbaren Anschluß an ein Deutschland durchzuführen, dessen Dioskurenbedürfnis sich in solch unleidlicher Kuppelung manifestiert" (S. 12) wie der zwischen Goethe und Schiller, und erkennt, daß Grillparzer "im öffentlichen kulturpolitischen Diskurs" der Ersten Republik dazu diente, "die Bindung der österreichischen an die deutsche Kultur zu demonstrieren". ${ }^{39}$ Dieser Gedanke wird 1930, als die Anschlußidee zu den Zollunionsverhandlungen geführt hatte, in der Formulierung vom "Anschluß des österreichischen Klassikers Grillparzer an Goethe und Schiller" wieder aufgenommen ( $F$ 834-37, S. 4).

Politische und kulturpolitische Rahmenbedingungen bestimmen so die Aggressivität von Grillparzer-Feier mehr als die Gestalt des Gefeierten; dessen Abwertung ist in beträchtlichem Ausmaß ein rhetorisches Mittel in der Auseinandersetzung mit den Grillparzer-Verehrern und ihren Absichten. Im Grunde

37. Rudolf Kassner, "Grillparzer (1954)." In: RK: Sämtliche Werke 10. Pfullingen 1991. S. 220-234. Hier S. 221.

38. Vgl. die Berichtigung der Stadt Wien zu Kraus' Äußerungen, in: F 595-600 (1922) S. 83-88.

39. Juliane Mikoletzky, "Vom Zensuropfer zum Klassiker. Grillparzer als österreichischer Nationaldichter." In: Grillparzer oder Die Wirklichkeit der Wirklichkeit. Bernhard Denscher, Walter Obermaier (Hrsg.) [Ausstellungskatalog]. Wien 1991. S. 135-141. Hier: S. 140. 
ist der Artikel von 1922 nur eine Umkehrung der bisherigen Position Kraus' gegenüber Grillparzer: hatte er ihn mindestens bis 1914 als selbstverständliches Element des kulturellen Milieus des von ihm wo nicht bejahten, so mindestens nicht grundsätzlich angegriffenen Staates angesehen und daher, bei aller Interesselosigkeit im Detail, seine Gültigkeit als Bildungsgut nicht in Zweifel gezogen, so lehnte er ihn nun ab, da er durch den Krieg auch das ehemalige Österreich-Ungarn abzulehnen gelernt hatte.

Was Kraus im einzelnen an "Österreichs Klassiker für die reifere Jugend" ( $F$ 724-25, 1926, S. 37) - auch hier wird wie im Wort "Taferlklassiker" in diskriminierender Absicht österreichische Schul-Terminologie gebraucht auszusetzen hatte, wird in Grillparzer-Feier nicht so recht deutlich. Er spricht von "papierner Ebenheit der Welt Grillparzers" (S. 12), von seiner "Blutleere", vom Mißlingen seines Versuchs, "den Himmel Griechenlands über dem Wienerwald zu wölben." Seine Epigramme seien "Einfall ohne Durchbruch" (S. 12) - wobei die im weiteren Verlauf des Beitrags aus einem Zeitungsaufsatz zitierten GrillparzerEpigramme zwar mit allgemeinen Bemerkungen wie "ganz nett" oder auch "ganz leer" (S. 19) bewertet werden, aber doch nicht so sehr jenes Urteil belegen als den (nicht genannten) Journalisten bloßstellen sollen; der Schwerpunkt dieses Teils der mehrere relativ selbständige Abschnitte umfassenden Grillparzer-Feier liegt darauf zu zeigen, daß die von jenem Journalisten zitierten Epigramme alles eher sind als Belege für seine in dem Artikel formulierten Behauptungen über Grillparzer. Die Überkommenheit seiner lyrischen Sprache, "die mittlere Kultur eines vorhandenen, unerschaffenen Ausdrucks" (S. 12), Mittelmäßigkeit und Bildungsdichtertum werden ohne nähere Begründung als Hauptvorwürfe gegen den Autor vorgebracht. Auch vom Aufbau des langen ersten Absatzes der GrillparzerFeier her, in dem die Kritik am Autor selbst konzentriert ist, bleiben als zentrale Angriffspunkte das "unterduckende Gemüt", belegt mit einem wörtlichen Zitat aus Der Traum ein Leben (S. 13), und die unzulässige Bevorzugung des "Bildungsdichters", des scheinbaren 'Klassikers' Grillparzer, vor den zwar nach den Kriterien von "Stoff" und "Form" weniger distinguierten, aber durch "Kraft" und "Wesen" überlegenen Autoren "volkstümlicherer Färbung": Raimund und Nestroy, wiewohl "an dessen Gebiet außen weder des Meeres noch der Liebe Wellen anschlagen." (S. 13)

Im Verlauf der Jahre gibt es Ansätze zu einer genaueren Begründung des Urteils über Grillparzer, in dem man auch ein wenig die Fortsetzung einer vielleicht doch schon auf die Schulzeit zurückgehenden, aber lange nicht genau überprüften Abneigung Kraus' gegen den Dichter erblicken mag. 1925 nimmt er auf die, von ihm aus einem Zeitungsartikel entnommene bekannte Tagebuchaufzeichnung: "Ich bin ein dorischer Dichter. Ich kümmere mich den Henker um die Sprache der Leipziger Magister und des Dresdener Liederkreises." 
(Tgb. 1625, 1828; HKA II/8, S. 296) Bezug und schreibt (F 679-85, 1925, S.

81):

Das glaube ich auch, und wenn jemand die Randstriche ansieht, die ich, der nicht gerade in dem Verdacht steht, die Sprache der Leipziger Magister und des Dresdener Liederkreises erfreulich zu finden, mir kürzlich in "Medea" und "Der Traum ein Leben" so ziemlich zu jedem Vers eingezeichnet habe, dann würde er zwar nicht genau wissen, der wievielte Klassiker der deutschen Nationalliteratur Grillparzer war, aber immerhin finden, daß es mir wie nur einem perfekten Literarhistoriker gelungen ist, den Einfluß des Dorischen bei Grillparzer nachzuweisen. (Freilich in der auffallenden Distanz gesehen, in der diese in den schlimmsten Zufallsversen gymnasialen Dilletantismus [!] verfaßten wirksamen Theaterstücke zu der sprachlich solideren Produktion etwa des "Treuen Dieners" stehen.)

1924/25 also hat Kraus einige Dramen Grillparzers auf ihre sprachlichen Qualitäten hin durchgesehen - und sie nicht für gut gefunden.

Spuren dieser Durchsicht, allerdings anderer Stücke, finden sich in zwei Glossen des Jahres 1924. Verse aus dem Bruderzwist in Habsburg (III. Akt. V. 1697ff.), die Kraus mit antihabsburgischer Intention aus der Reichspost zitiert, nennt er "nicht sehr gewichtig" und weist trotz der anderen Zielrichtung der Glosse vor allem metrisches Mißlingen nach, indem etwa ein (fünfhebiger) Blankvers zum (sechshebigen) Alexandriner geworden ist und in einem anderen aus metrischen Gründen die Satzglieder so umgestellt worden sind, daß der Sinn in sein Gegenteil verkehrt erscheint ("H. H."; F 657-67, 1924, S. 53f.).

Die Wendung, im gleichen Heft der Fackel (S. 200f.) erschienen, bezieht sich vornehmlich auf einen bekannten Vers aus dem Ottokar (III. Akt; V. 1671f.):

[...] es ist ein gutes Land,

Wohl wert, daß sich ein Fürst sein unterwinde!

Obwohl auch diese Glosse bezeichnenderweise mit einer antimonarchischen Pointe endet, kommt es Kraus hier doch vor allem auf den falschen, katachretischen Gebrauch von "unterwinden" an, der genau analysiert wird, als Beispiel für die Arbeitsweise eines "Bildungsdichters", der "infolgedessen sich beim Dichten auf einen so trügerischen Besitz wie die Bildung verließ, mit der man oft etwas zu erlangen wähnt, was man dann doch nicht hat." Die zitierte Stelle sei "das Musterbeispiel einer Wendung, die erlesen ist, aber falsch erlesen, die schon stimmen wird, aber nicht stimmt, [...]" (S. 200).

Die Wurzeln dieser sprachlichen Kritik an Grillparzer sind in Kraus' literarischem Traditionsbewußtsein zu sehen. Er dachte im Sprachlichen wie im Literarischen durchaus normativ, und den beiden für ihn verbindlichen Normen 
genügte Grillparzer nicht. Die eine Norm, bestimmend für große Teile von Kraus' Satire, war die des Wiener Volkstheaters, die bei Grillparzers mindestens in der Sprache seiner Dramen kaum einen Niederschlag gefunden hat; die andere, von welcher der 'pathetische' Teil von Kraus' Werk - Teile der Lyrik, die überhöhenden Szenen der Letzten Tage der Menschheit, stark mit Mitteln der traditionellen Rhetorik gestaltete Passagen der Satiren und Polemiken - ausgeht, war von der Goethe-Zeit geprägt, vornehmlich vom klassischen Goethe selbst und von der Schlegel-Tieckschen Shakespeare-Übersetzung. Grillparzer schien nun dieser Tradition verpflichtet, vor allem auch durch sein Festhalten am Vers, hat sie aber durch eine gewisse Skepsis gegenüber der Sprache auch schon wieder aufgelockert und durchbrochen. Für solche Abweichungen von der klassischen Sprach-Norm, wie sie sich ganz explizit auch bei Heine findet, hatte Kraus keine Toleranz ${ }^{40}$; obwohl er sie bei Grillparzer, anders als im Fall des wirklich gehaßten Heine, offensichtlich mehr geahnt als analysiert hat, ist der sprachkritische Aspekt seines Urteils über den Wiener Dramatiker durchaus in Parallele zur Heine-Polemik zu sehen (allerdings ganz ohne deren antijournalistische Aspekte). Zudem war Kraus' Auffassung des Theaters so stark von der Sprache bestimmt, daß er für die Rolle von Bild und Gestik bei Grillparzer wohl nur wenig Sinn hatte.

Neben den wenigen Stellen, an denen in der Fackel der Versuch gemacht wird, Schwächen von Grillparzers Sprache nachzuweisen, steht gelegentlich der Vorwurf, der Dramatiker habe "die Dämonen eines Traumes in Zucker" eingemacht ( $F$ 724-25, 1926, S. 37) - anders als Kraus in Traumtheater (1924) und vor allem in Traumstück (1923); er habe keinen wirklichen Sinn für Tragik gehabt, habe zu leicht Kompromisse geschlossen. Solche Formulierungen konkretisieren wohl die Behauptung von der "papiernen Ebenheit der Welt Grillparzers" in Grillparzer-Feier (S. 12). Wortspielhaft ist dieses Urteil bezeichnenderweise - in Nestroy und das Burgtheater im Nebensatz zu einer Bemerkung über den Schauspieler Thaller formuliert ( $F$ 676-78, 1925, S. 14); dieser habe "bloß einen Lebenstypus dargestellt",

der weder in Humor noch in Tragik die geringste Verbindung mit dem schöpferischen Theaterwesen hatte, etwa den grantig-gemütlichen Rechnungsrat, der vom Grillparzer etwas Grill abbekommen hat [...]

Im Wortspiel kommt es selbstverständlich weniger auf den damals ohnehin kaum besonders geläufigen 'Grill' als vielmehr auf die Absenz der für die Tragödie wichtigen Parzen an.

Diese negativen Urteile, die die Grillparzer-Philologie durchaus zum

40. Vgl. Mechthild Borries, Ein Angriff auf Heinrich Heine. Kritische Betrachtungen zu Karl Kraus. Stuttgart 1971 (=Studien zu Poetik und Geschichte der Literatur 13). Besonders S. $61 \mathrm{ff}$. 
Nachdenken anregen können, zur Falsifikation herausforden sollen, will ich aus methodischen Gründen nicht auf ihre Richtigkeit hin untersuchen. Denn eine solche Überprüfung würde, entgegen dem eigentlichen Sinn der Rezeptionsforschung, das Vorhandensein einer 'richtigen Interpretation' voraussetzen. Rezeptionsforschung soll aber Rezeptionszeugnisse analysieren und zu beschreiben versuchen, wie es zu einer bestimmten Lektüre von Werken kommt; sie soll den Rezeptionszeugnissen aber nicht Zensuren geben.

$\mathrm{Da} B$ die kritische Grillparzer-Rezeption von Kraus wiederum rezipiert worden ist, ist hingegen kurz zu erwähnen. Das sehr kritische Grillparzer-Bild der wichtigsten Literaturkritiker in den Anfängen der Zweiten Republik ${ }^{41}$ hängt gewiß damit zusammen, daß sie, insbesondere Torberg und Weigel, genaue KrausLeser gewesen sind. ${ }^{42}$ Und die deutliche Akzentuierung dieses negativen Urteils ist wiederum durch den massiven Versuch der Kulturpolitik der Zweiten Republik provoziert worden, Grillparzer zum Staatsdichter Österreichs zu machen, was etwa auch zur Wahl des Ottokar für die Eröffnungsvorstellung im wiederaufgebauten Burgtheater (1955) geführt hat. Nichts verstellt ja bis heute meiner Generation den Zugang zu Grillparzer so sehr, wie diese Stilisierung des Autors zum 'Klassiker' Österreichs, gerade auch in der Schullektüre. (Handkes Rede beweist allerdings ebenso wie Aussagen von Ingeborg Bachmann ${ }^{43}$, Barbara Frischmuth und anderen, daß es in der Literatur Österreichs nach 1945 durchaus auch Zustimmung zu Grillparzer gibt, nicht nur bei den Repräsentanten der offiziösen Kulturpolitik).

Nach diesem kleinen Exkurs über das Fortwirken sowohl von Kraus' Grillparzer-Kritik wie der Bedingungen, unter denen sie entstanden ist, zurück zu ihr selbst.

Sie ist nämlich in wesentlichen Zügen zu modifizieren: nirgends ist Kraus' Kritik totale Verdammung; viele der hier zitierten Fackel-Stellen verbinden sich mit partieller Anerkennung von Grillparzers Leistung. Wo die "Zufallsverse" der Medea getadelt werden, wird doch die "sprachlich solidere Produktion" des Treuen Dieners und werden selbst die getadelten Dramen als "wirksame Theaterstücke" anerkannt ( $F$ 679-85, 1925, S. 81), was bei Kraus freilich nicht unbedingt als literarisches Lob anzusehen ist; eine analoge Stelle aus GrillparzerFeier (S. 12) habe ich bereits zitiert.

41. Von einer aus Anhängern von Kraus gebildete Fronde gegen Grillparzer schon in der Ersten Republik spricht Kassner (Anm. 37)

42. Vgl. Sigurd Paul Scheichl, "Die Stellung der österreichischen Literatur des 19. Jahrhunderts im literarischen Leben der Gegenwart". In: Traditionen in der neueren österreichischen Literatur. Friedbert Aspetsberger (Hrsg.). Wien 1980. S. 5-30. (=Schriften des Institutes für Österreichkunde 37). Zu Grillparzer besonders S. 9-14.

43. Vgl. ebenda, S. 11. 
Selbst in dieser ist dem Tadel an der "überkommenen" Sprache von Grillparzers Lyrik die Anerkennung beigegeben, sie sei immerhin "gut gehalten" (S. 12). "Vornehmheit, Würde und Feinheit der formalen Bildung" (S. 13) werden dem Autor ebenso zugestanden wie "vereinzelte Schönheiten" (S. 14), und Grillparzers "Lebenswürde, die alle Öffentlichkeit mied" (S. 14), wird die Bereitschaft heutiger Autoren kontrastiert, sich jederzeit auf Wunsch der Zeitungen (und gegen Honorar) zu allem Möglichen zu äußern. Bei der Bedeutung der Pressefeindschaft als Thema Kraus' bedeutet gerade diese Einschränkung nicht wenig.

1925 hat Kraus, wie oft, einen Artikel über sein Werk in der Fackel nachgedruckt; dessen Autor, der von Kraus geschätzte tschechische Germanist Otokar Fischer ${ }^{44}$, stellt darin einen Zusammenhang zwischen dem Satiriker und Grillparzers Epigrammatik her $\left(F\right.$ 649-54, 1926, S. 89).$^{45}$ Kraus erhebt gegen diesen Vergleich weder Einspruch noch Protest, die bei vergleichbaren Aussagen über Parallelen zwischen seinem Werk und dem Heines ganz gewiß nicht ausgeblieben wären. Auch hier also eine indirekte Spur von Anerkennung.

Und noch im letzten Heft der Fackel drückt Kraus neben fortdauernder Distanz auch "Hochschätzung vor Grillparzers antibanalem Denken" aus ( $F$ 91722, 1936, S. 73).

$\mathrm{Zu}$ diesem antibanalen Denken gehört für ihn zweifellos vor allem Grillparzers Presse-Kritik, die in der Fackel zuerst von Karl Bleibtreu erwähnt worden ist (F 298-99, 1910, S. 49). Es ist denkbar, daß Kraus durch diesen Beitrag seines damaligen Mitarbeiters auf das Gedicht Der Henker hole die Journale von 1844 (HKA I/11, S. 191) gestoßen ist, das er wenig später mit einem aktualisierenden Titel in seiner Zeitschrift abgedruckt hat. ${ }^{46}$

Das Gedicht stellt er dann mit einer ironischen Vorbemerkung auch ans Ende von Grillparzer-Feier: auch er, Kraus, wolle den österreichischen Dichter feiern, "um einiger Strophen willen, die bedauerlicherweise bei der Feier der Concordia von Herrn Reimers nicht gesprochen worden sind" ( $F$ 588-94, 1922, S. 21) $)^{47}$ :

44. Zu diesem vgl. Jaromír Loužil und Zdeněk Šolle, "Karl Kraus und die Tschechoslowakei." In: Kraus-Heft 15 (1980). S. 1-8; Jaromír Louzil, "Ein Nachtrag zum Thema Karl Kraus und Otokar Fischer." In: Kraus-Heft 33 (1985).S. 2f.

45. Otakar [!] Fischer, "Poslední dnové lidstva." In: Ceské Slovo 15 (1923). Nr. 290 (12. Dezember). S. 2f. Zitiert nach Otto Kerry, Karl-Kraus-Bibliographie. München 1970 (K 180).

46. Das Auftauchen des Motivs des Henkers ("daß hoch hinauf der Henker / euch bringt!") im Couplet des Schwarz-Drucker (S 9, S. 412f.) könnte eine Anspielung auf dieses Epigramm Grillparzers sein.

47. Die Concordia war eine Vereinigung liberaler Journalisten und Publizisten; Georg Reimers war ein von Kraus wenig geschätzter Schauspieler des damaligen 
Der Henker hole die Journale,

Sie sind das Brandmal unsrer neuen Welt,

Der ekle Abhub von dem Wissenmahle,

Der, für die Viehmast, in die Zuber fällt.

$[\ldots]$

Das Zitat an dieser Stelle bestätigt das Verständnis von Grillparzer-Feier als einem Text, der viel mehr als den Gefeierten die Feiernden treffen will, nicht so sehr weil sie aus dessen Werk das immerhin vorhandene Widerständige verschwinden lassen, sondern vor allem weil sie Journalisten oder doch Preßhörige sind, während Grillparzer sich in die Reihe der preßfeindlichen Ahnen der Fackel einordnen läßt.

Diesem epigrammatischen Gedicht ist auch zweimal ${ }^{48}$ die Auszeichnung der Aufnahme in Kraus' Vorlesungen widerfahren, allerdings nur relativ bald nach dem ersten Abdruck in der Fackel: im Rahmen einer Lesung von Werken der Mitarbeiter der Zeitschrift (V 14, 15. Mai 1911) ${ }^{49}$ - unter dem Titel Die Presse - und als Schluß der Einleitung zu Kraus' zweitem Nestroy-Abend im Jahr 1912 (V 26, 23. Mai 1912; F 351-53, 1912, S. 39f.), wo also Nestroy und Grillparzer noch keineswegs gegeneinander ausgespielt wurden. Für das von Grillparzer-Feier bestimmte Bild des Grillparzer-Verständnisses von Kraus ist es bezeichnend, daß Christian Wagenknecht in seiner jüngst erschienenen schönen Anthologie der von Kraus öffentlich vorgetragenen Gedichte diese Verse nicht aufgenommen hat ${ }^{50}$, obwohl sie "der erste nichtzeitgenössische Text" waren, den Kraus in das Programm einer seiner Vorlesungen aufgenommen hat ${ }^{51}$ - freilich nicht so sehr wegen seiner ästhetischen Qualitäten als wegen seiner Integrierbarkeit in Kraus' Polemik gegen die Presse.

Auch ein anderes, auf Saphir gemünztes pressefeindliches Epigramm (Fehlgeburt, 1840; HKA I/12, S. 112) wird in der Fackel schon 1902 zitiert, in einem Beitrag ( $F 111$, S. 29f.), der von einem Mitarbeiter stammen dürfte, und Kraus spielt darauf noch 1922, mit völlig neutraler Nennung Grillparzers, an ( $F$

\section{Burgtheaters.}

48. Dazu wahrscheinlich noch ein drittes Mal im Rahmen der Lesung von GrillparzerFeier (V 219, 22.1.1922).

49. Zählung der Vorlesungen nach Christian Wagenknecht: Die Vorlesungen von Karl Kraus. Ein chronologisches Verzeichnis. In: Kraus-Heft 35/36 (1985). S. 1-30.

50. Lyrik der Deutschen für seine Vorlesungen ausgewählt von Karl Kraus. Hrsg. von Christian Wagenknecht. München 1990. Die Auslassung der Grillparzer-Verse wird dort auch nicht gerechtfertigt. Insofern ist auch Wagenknechts Aussage (Nachwort, ebd, S. 173), Kraus habe mit einer Ausnahme "kein eigentlich epigrammatisches Gedicht" eines anderen Autors vorgetragen, zu modifizieren.

51. Krolop (Anm. 7), S. 330, Anm. 70. 
608-12, 1922, S. 79), allerdings in einem Zusammenhang, in dem tatsächlich von Saphir die Rede ist: Kraus weist einen Vergleich seines Werkes mit diesem zurück.

Im Bereich der Epigrammatik ${ }^{52}$ scheint Kraus also trotz dem abwertenden Urteil in Grillparzer-Feier eher Zugang zum Autor zu finden, insbesondere aus inhaltlichen Gründen, wegen der pressefeindlichen Stellen, die er wohl auch mit dem "antibanalen Denken" meint.

Es liegt mir fern, angesichts des Grillparzer-Jubiläums den Versuch einer Abmilderung der kritischen Urteile Kraus' über Grillparzer zu machen. Daß Kraus sich in einem eher zufälligen Zusammenhang heftig dagegen verwehrt, daß der Verlag Schroll versuchte, die Fackel nicht nur als Vehikel der Werbung für die Nestroy-, sondern auch für die Grillparzer- und die Anzengruber-Ausgabe zu nützen (F 676-78, 1925, S. 42), ist zweifellos Ausdruck eindeutiger Abwertung eines Autors, dessen durch Journalisten und Literaturhistoriker verschuldete Überschätzung ihm dringend der Korrektur $\mathrm{zu}$ bedürfen schien. Die Einschränkungen des negativen Urteils, auch durch das achtungsvolle Schweigen der Jahre vor 1922, dürfen dennoch nicht übersehen werden. In einer Zeit, in der Nestroy auch öffentlich jene Ehren erwiesen werden, die er zweifellos verdient, würde möglicherweise Kraus' durch den Kontrast bestimmtes Urteil über Grillparzer auch anders ausgefallen sein, wäre Grillparzer-Feier vielleicht so nicht geschrieben worden.

Zum Abschluß Anekdotisches: 1914 erhielt Kraus von Sidonie Nádherný und ihrem Bruder das Autograf einer Stammbucheintragung Grillparzers ("Den du beklagst ..." $)^{53}$ zum Geschenk, das er, mit Angabe der Spender, in der Fackel als Faksimile veröffentlicht hat $(F$ 400-03, 1914, S. 96; HKA I/12, S. 314). Das Manuskript der an sich wenig bedeutenden Verse hing bis zu seinem Tod in Kraus' Arbeitszimmer. ${ }^{54}$ Das wäre bei allem bildungsbürgerlichen Stolz auf ein Dichtermanuskript und bei allem sentimentalem Wert, den dieses Geschenk für ihn haben mußte, denn doch nicht denkbar gewesen ohne einen Rest von Ambivalenz des Urteils gegenüber Grillparzer, ohne die neben der Kritik an seiner Sprache doch auch vorhandene Hochachtung vor dem Menschen Grillparzer und seinem "antibanalen Denken".

Dessen Anerkennung ist vielleicht nicht zufällig das letzte Wort der Fackel über Franz Grillparzer.

52. Vgl. auch $F 15,1899$, S. 16, mit dem Zitat des Grillparzer-Epigramms "Göthe" (HKA I/12, S. 93) gegen Hugo von Hofmannsthal.

53. Zur nicht geklärten Herkunft des Manuskripts vgl. $H K A \mathrm{I} / 12,2$ (Anmerkungsteil) S. 380. Die historisch-kritische Ausgabe hat den Text übrigens nach dem Faksimile in der Fackel aufgenommen.

54. Vgl. die Beschreibung dieses Raumes bei Leopold Liegler, "Meine Erinnerungen an Karl Kraus." In: Kraus-Heft 25 (1983). S. 1-18. Hier: S. 11. 